\title{
VIKA - Konzeptstudien eines virtuellen Konstruktionsberaters für additiv zu fertigende Flugzeugstrukturbauteile
}

Johann Steffen

Gegenstand der Arbeit ist die konzeptionelle Ausarbeitung einer virtuellen Anwendung, die es den Anwendern in der Flugzeugstrukturkonstruktion im Kontext der additiven Fertigung ermöglicht, interaktiv und intuitiv wichtige Entscheidungen für den Bauteilentstehungsprozess zu treffen. Dabei soll sich die Anwendung adaptiv je nach Anwendungsfall in der Informationsbereitstellung an die jeweils benötigten Anforderungen und Bedürfnisse des Anwenders anpassen können.

Keywords: Additive Fertigung, Konstruktionsberatung, multikriterielle Entscheidungen, Entscheidungsunterstützungssystem, Implizite Wissensrepräsentation

\section{Einleitung}

„Die additive Fertigung ist eine aufstrebende Technologie, die zahlreiche dreidimensionale Druckprozesse (3D Druck) zum schichtweisen Verbinden von Materialien umfasst, um Bauteile werkzeuglos aus Konstruktionsdaten zu produzieren" (Serdar, 2016). Durch diese Technologie können komplexe Formen, Multimaterial- und Multifunktionsbauteile in einem Arbeitsgang hergestellt werden, was einen großen Vorteil gegenüber konventionellen Fertigungsprozessen darstellt (ASTM, 2009). In den letzten zwei Jahrzehnten hat die Forschung bedeutende Fortschritte bei der Entwicklung innovativer Fertigungsprozesse, wie zum Beispiel dem selektiven Lasersintern und Laserschmelzen erzielt (Pahl, Beitz, Feldhusen, Grote, 2007). Der zunehmende Einsatz von additiven Fertigungstechnologien in der Luft- und Raumfahrt, Automobil-, Biomedizin- und anderen Industrien hat zu einer erhöhten Nachfrage nach qualifizierten Fachkräften geführt, die alle Aspekte der Produktentstehung beherrschen müssen. Um diesem Bedarf gerecht zu werden, benötigen die Anwender der Technologie fortgeschrittene und fundierte Fachkenntnisse im Umgang mit CAD, CAE und CAM-Systemen, die speziell auf die Entwicklung von additiven Prototypen- und Serienbauteilen zugeschnitten sind und die sich auf die Konstruktion von 3D-Strukturen mit einer wachsenden Anzahl von Metall-, Kunststoff- und anderen Werkstoffen konzentrieren (Agarwala, Chin, 2015). 
Die additive Fertigung ist charakterisiert durch eine neue Herangehensweise in der Auslegung der Bauteile. Die Modellierung der Geometrie ist nicht mehr restriktiv an Werkzeugformen geknüpft und folglich lassen sich Leichtbaupotentiale z.B. durch topologieoptimierte Gestaltungsstrategien ohne größere Folgekosten herstellen. Mit der additiven Fertigung sind zudem effektive Rationalisierungen in der Baugruppenauslegung möglich, die sich aus Funktionsintegrationen herleiten lassen.

Resultierend wächst auch das Interesse der Luftfahrtindustrie weiter, additiv gefertigte Bauteile für den Flugbetrieb einzusetzen. Für einen wirtschaftlich erfolgreichen Einsatz der Technologie ist immer mehr erfahrungsbasiertes Spezialwissen erforderlich. Die inzwischen zahlreichen erschienenen Fach- und Lehrbücher sind zu unspezifisch, um als Anleitung für die Konstruktion zu dienen. Das Fachwissen in der additiven Fertigung ist zudem im letzten Jahrzehnt hochgradig dynamisch angewachsen und in Printals auch digitalen Medien sehr diffus verteilt. Auch die Aktualität und Validierung dieser agilen Informationsdarstellung kann vor allem nicht immer sichergestellt werden.

Dieser Umstand stellt die Anwender und weitere Stakeholder der Luftfahrtindustrie mit unterschiedlichen Wissensständen und Fragestellungen aktuell noch vor die enorme Herausforderung, das benötigte Wissen zeiteffizient zu selektieren und zu validieren, um die richtigen Entscheidungen für den gesamten Entwicklungsprozess (z.B. Anwendungsfälle, Verfügbarkeit und Robustheit von Konstruktionsregeln/-richtlinien, Material-, Maschinenauswahl, usw. ...) zu treffen.

Das Forschungsvorhaben VIKA befasst sich folglich mit einer wissenschaftlich methodischen Herangehensweise, die oben genannte Problematik in eine konzeptionelle Lösung zu überführen, indem konkrete Vorschläge für die technische Realisierung erarbeitet, prototypisch umgesetzt und bewertet werden. Ziel ist es, eine klare Verwertungsperspektive für die spätere Weiterentwicklung der Anwendung zu schaffen, sodass aus der Konzeptstudie eine relevante Anwendung für die Luftfahrtindustrie abgeleitet werden kann.

\section{Methodische Herangehensweise, Problemstellung und Abgrenzung}

Zunächst wurden frei zugängliche bzw. verfügbare Softwarelösungen und Unterstützungssysteme, die sich mit der virtuellen Produktentwicklung additiver Bauteile befassen, angewandt, analysiert und in diesem Kontext der Stand der Forschung erarbeitet.

Hierbei konnte festgestellt werden, dass sich der virtuelle Bauteilentstehungsprozess additiver Bauteile stark gewandelt hat: In der Konstruktion konventionell gefertigter 
Bauteile sind die jeweiligen Disziplinen Konstruktion, Berechnung und Fertigungs-vorbereitung und Durchführung in unterschiedle Anwendungen verteilt, die von Akteuren genutzt werden, die in dem jeweiligen Fachgebiet über das dazugehörige Spezialwissen verfügen.

In der additiven Fertigung hingegen wird aufgrund der werkzeuglosen Herstellung der Bauteile oftmals angestrebt, die virtuelle Prozess- und Lieferkette durchgängig zu halten, um die Herstellungs- und Entwicklungskosten zu reduzieren. Die Übergänge und Grenzen der Domänen Design, Berechnung und Absicherung der Ergebnisse werden aufgelöst und fließen somit ineinander über.

Folglich sehen sich die Anwender in der virtuellen Produktentwicklung jetzt vor die Herausforderung gestellt, ihre Kompetenzen und ihr Fachwissen mehr auf der kognitiven und disziplinübergreifenden Ebene auf- bzw. auszubauen, um den Gestaltungsprozess zielorientierter durchzuführen.

Die methodische Herangehensweise an eine Konstruktion für ein additiv zu fertigendes Bauteil grenzt sich dabei von alt bekannten Methoden der Bauteilgestaltung ab: Entgegen der computergestützten Erzeugung von Geometrien, die auf den Fähigkeiten und dem Können des Konstrukteurs beruhen, ist der Konstrukteur darauf bedacht, die automatisiert generierten Topologien unter Beachtung von Herstellbarkeit und der Nachweisführung in der mechanischen Festigkeit zu bewerten. Da die Prozesse in der additiven Fertigung oftmals noch sehr instabil sind, Bauteileigenschaften erst bei der Herstellung entstehen und die Reproduzierbarkeit der Bauteile auf bereits zertifizierte Materialien, Maschinen, Geometrien oder gesamten Prozessen basiert, ist es notwendig, Wissen über diese wichtigen Entwicklungsparameter für die Anwender zeitnah und fallbasiert zur Verfügung zu stellen.

Viele Konstrukteure arbeiten mit proprietären Referenzen und eigens aufgebauten Methodenwissen und abgeleiteten Konstruktionsregelkatalogen, die nur intern von einem kleineren Anwenderkreis vermittelt und weitergegeben werden. Das Wissen ist somit an spezielle Wissensorte und Personen gebunden und ist begrenzt verfügbar. Novizen fehlt es gänzlich nach einer Möglichkeit, auf bestehendes Expertenwissen und Methoden zurückzugreifen und somit eine additive Konstruktion auf Plausibilität und Validität zu überprüfen.

Um diesen Paradigmenwechsel in der additiven Bauteilkonstruktion zu unterstützen, wurden in den letzten Jahren unzählige Softwarelösungen geschaffen, die eine durchgängige und disziplinübergreifende virtuelle Prozesskette ermöglichen. 
Es werden in den Anwendungen zwar Möglichkeiten geschaffen, Bauteile intuitiv zu gestalten, Topologien automatisiert zu erzeugen und die Druckprozesse zu simulieren und auch quantitativ sowie qualitativ auszuwerten - jedoch wird dabei auf eine integrierte Wissensbasis mit Experten- und Methodenwissen gänzlich verzichtet. Die Anwender werden oftmals vor die Frage nach dem „Wie?" gestellt, wobei die Antworten auf die Fragen „Was" und "Womit?" bereits aufgelöst sind.

Aus den ersten Voruntersuchungen konnte final abgeleitet werden, dass aufgrund der unerschöpflichen Anzahl an existenten Softwarelösungen eine Ausarbeitung einer spezifisch abgestimmten CAD-, CAE- oder CAM-Schnittstelle für eine Softwarelösung unter Einbezug der KBE-Methode (Knowledge based engineering) für die virtuelle Konstruktionsberatung kategorisch auszuschließen und somit nicht zielführend ist.

Die oben genannte Annahme wird zudem noch durch die These gestützt, dass sich der Markt der Softwareanwendungen stets dynamisch weiterentwickelt und somit eine konzipierte Systemschnittstelle den Anwenderkreis durch Lizenzbestimmungen, Einarbeitung in die jeweilige Softwarelösung und Akzeptanz sehr stark eingrenzt. Eine Ausarbeitung diverser CAx-Schnittstellen würde den Rahmen des Forschungsprojekts sprengen. Eine Umsetzung einer universalen CAx-Schnittstelle ist programmier-technisch zudem nicht möglich, da jede Lösung mit eigenen Algorithmen, Funktionen und Systemstrukturen arbeitet.

Ziel ist es daher, mit den Methoden des Systems-Engineerings und der damit verknüpften modellbasierten Denkweise eine übergreifende digitale Lösung für die Repräsentation von Methodenwissen in der additiven Fertigung zu entwickeln, die die Anwender neben der konstruktiven Arbeit berät und adaptiv und sequenziell im Entscheidungsfindungsprozess unterstützt. Nur dieses Wissen befähigt die Anwender alleine dazu, Heuristiken zu erlernen, um im Gestaltungsprozess die richtigen Annahmen und Entscheidungen zu treffen.

Die Anwendung soll benutzerfreundlich aktualisierbar und erweiterbar sein, sodass konsensbasiertes und relevantes Wissen für die Bearbeitung von additiven Konstruktionen unter Einbezug diverser Softwarelösungen abgebildet und abgerufen werden kann. Dabei erscheint es als wichtig, spezielles proprietäres Wissen geschützt und strategisch verfügbar zu machen. Um ein innovatives und auch nachhaltiges System zu erschaffen, ist es unabdingbar, sich wissenschaftlich mit dem gesamten Wissensmanagementprozess auseinander zu setzen. 
Zunächst galt es die potentiellen Nutzer von VIKA zu ermitteln. Entsprechend wurde eine umfangreiche Stakeholder- und Bedürfnisanalyse im Projekt durchgeführt (Abbildung 1).

Mit dieser Untersuchung aus der Fachdisziplin des Systems-Engineering und der Szenario-Technik wurden mit der Methode "Persona“ nach Cooper (Cooper, Reimann, Cronin, 2007) weitere denkbare Anwender-Persönlichkeitsprofile generiert (Abbildung 2). In der Systementwicklung nach dem „Top Down“-Prinzip konnten die möglichen Anwenderprofile mit den ermittelten Bedürfnissen und Anforderungen an eine virtuelle Beratungslösung in zahlreich generierte Anwendungsfälle mit möglichen Interaktionsszenarien in das System-Design transformiert werden (Abbildung 3).

Aus den Anwendungsfällen gehen wichtige Erkenntnisse für den Aufbau einer umfangreichen Funktionsliste hervor, die als Ausgangsbasis für die konzeptionelle Auslegung der virtuellen Anwendung dienen.

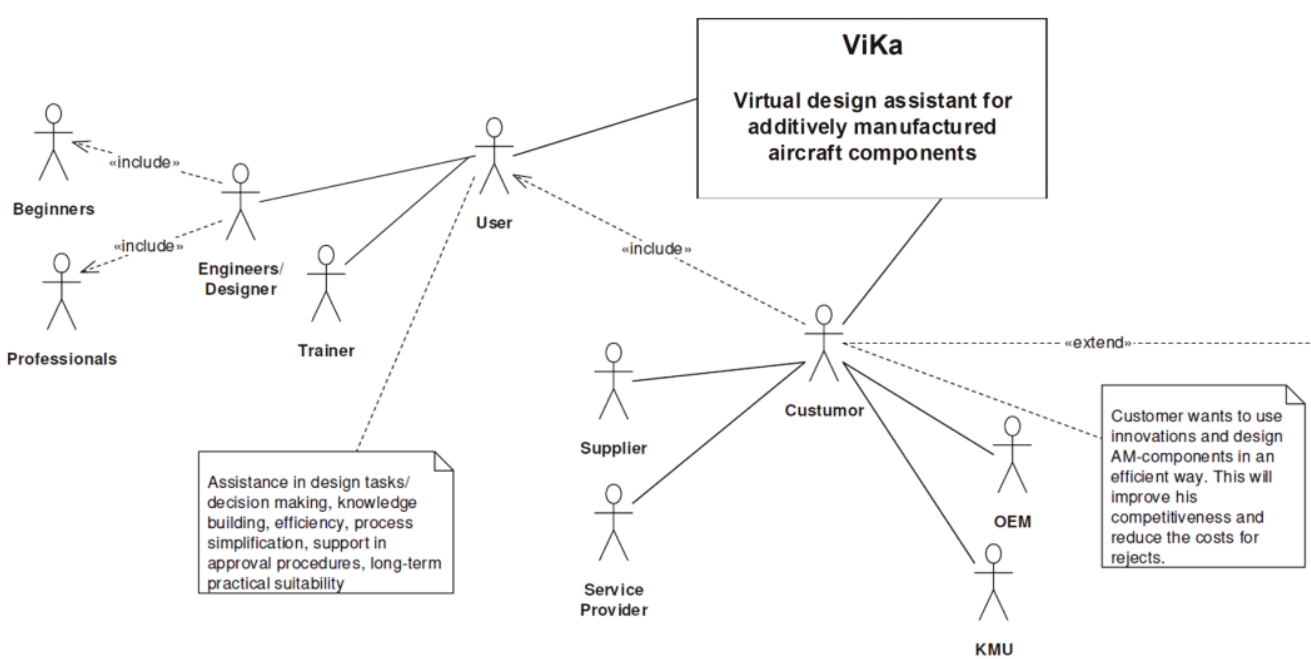

Abbildung 1: Auszug aus der Stakeholder Analyse (Anwender) 


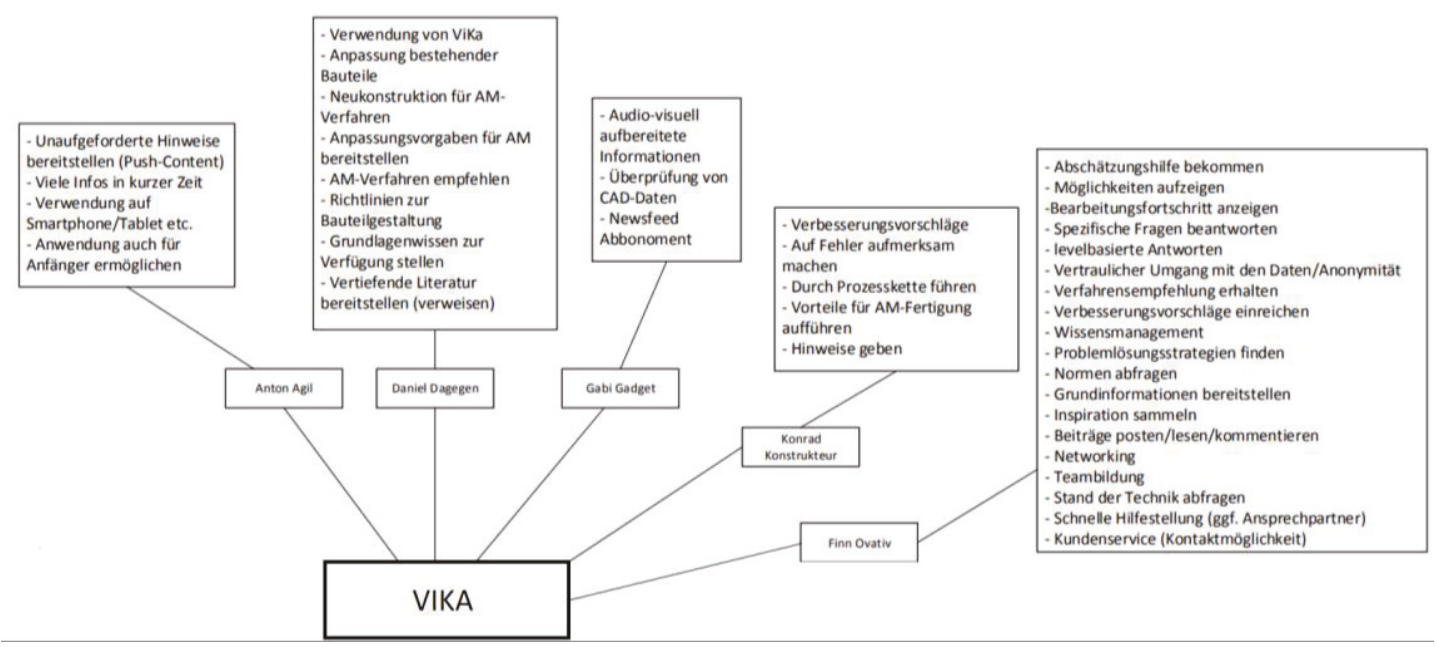

Abbildung 2: Auszug aus der Persona-Methode - Funktionen für VIKA

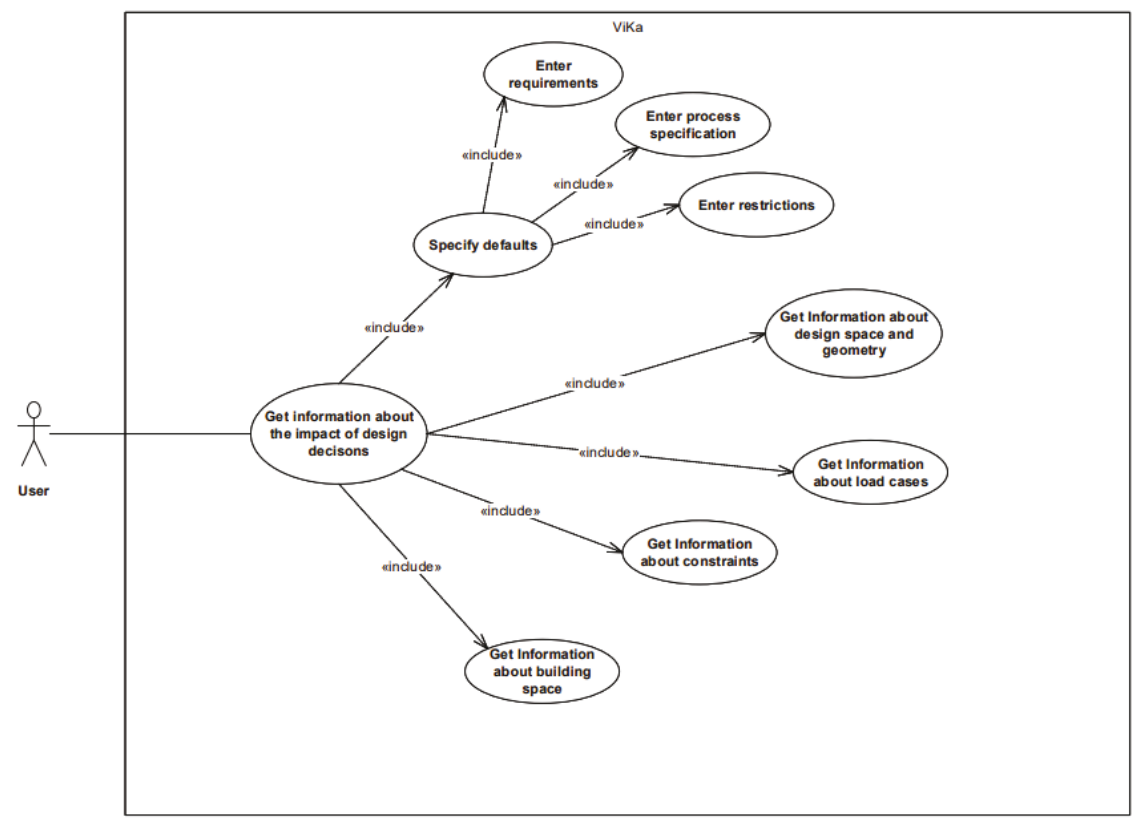

Abbildung 3: Anwendungsfallanalyse-Auswirkungen auf Design-Entscheidungen 


\section{Stand der Forschung}

In der Wissenschaft und Forschung ist ein sehr breites und unerschöpfliches Spektrum an Fachaufsätzen, Untersuchungen, Methoden und Werkzeugen für den Design-Entscheidungsfindungsprozess im Kontext der additiven Fertigung existent: So untersuchten Qi et al. in einem kategorischen Rahmenwerk die Formalisierung des additiven Fertigungsprozesses mit Hilfe von Ontologien, um grundlegende und allgemeine Kenntnisse über Design- und Prozessparameter, Regeln bzw. Richtlinien und deren gegenseitige Beeinflussung für eine Integration in CAx-Plattformen zu modellieren (Qi, Pagani, Scott, Jiang, 2018). Wang et al. schlagen im Kontext der geeigneten ProzessSelektion eine Hybridmethode aus dem sogenannten „Design-by-shopping“ Ansatz in Kombination mit einer modifizierten TOPSIS-Methode vor, bei dem der Benutzer in einer virtuellen Anwendung in einer interaktiven Weise seine Präferenzen für Prozessparameter selektiert, um über verschiedene Formalisierungs-methoden eine hierarchisch gestufte Reihenfolge von Lösungsansätzen und Vorschlägen zu erhalten (Wang, Zhong, Xu, 2018). Yao et al. untersuchten mit einer Fallstudie den Ansatz unerfahrenen Designern Empfehlungen, Konstruktions-merkmale und Entwurfslösungen mit Hilfe des maschinellen Lernens in Form von hybriden Algorithmen codierter Clustern bereitzustellen, die über bereits bestehende Anwendungsfälle trainiert werden können (Yao, Moon, Bi, 2017). Im Programm des European Union's Horizon 2020 wurde über das Projekt „Encompass“ ein integrierbares Design-, Entscheidungs- und Unterstützungssystem für die gesamte Prozesskette im Kontext des laserbasierten Pulverbettverfahrens (LPBF) entwickelt. Um die Leistungsfähigkeit des Systems zu demonstrieren, wurden Anwendungsfälle aus der Automobil-, Luftfahrt und Medizinindustrie herangezogen und unter den Aspekten „Time to Market“, Produktivität der Prozesskette und Auswirkungen auf die Produktionskosten ausgewertet (Helmrath, Catarino, Brackett, 2020). Eisman et al. schlagen ein Rahmenwerk für das Systemdesign von virtuellen Beratern in geschlossenen Wissensdomänen vor, indem eine webbasierte User-Client-Architektur mit drei Benutzermodulen erschaffen wird. Diese agieren mit Spracherkennung, aus dem isoliert Informationseinheiten extrahiert und verarbeitet werden. Ein Dialogmanager führt Aktionen basierend auf gruppierten Entscheidungsmustern und einer Ontologie-Datenbank aus. Ein Kommunikationsgenerator transferiert die Algorithmen in eine natürliche Sprachausgabe (Eisman, Lòpez, Castro, 2012). Hartmann erwähnt, dass regelbasierte XPS (Expertensysteme) über entsprechende Komponenten und Präsentationsformen verfügen und im Bereich der Ausbildung sogar einen Vorteil gegenüber menschlichen Experten besitzen, da im Problemlösungsprozess die Qualität der Wissensvermittlung sehr stark von den Befähigungen der Experten abhängig ist, Erfahrungen nachvollziehbar und verständlich zu 
erklären (Hartmann, 2015). Bei hochdynamischen Wissensbasen ist allerdings eine stetige Wartung und Pflege unerlässlich und im Hinblick der Wissensbereitstellung droht ein XPS-System zeitnah zu „veraltern“. Auch gilt es die Korrektheit und die Vollständigkeit stetig zu überprüfen, was enorme Ressourcen in Unternehmen bindet (Remus, 2002). Kratzer stellt fest, das Strukturen und der Aufbau von wissensbasierten Systemen sich stark an die jeweiligen Organisationsformen und Unternehmensprozessen orientieren müssen, damit eine passende Anwendung gefunden werden kann (Kratzer, 2014). Für die Entwicklung eines agentenbasierten Softwareberatungssystems ist ein Denken in Rollen und nicht in Personen zielführender. Es wird eine Differenzierung in die Rollen des Wissensingenieurs, des Entscheiders, des Konstrukteurs und Softwareingenieurs vorgegeben (Luft, Breitsprecher, Roth, 2012). Saue et al. schlagen ein XML-basiertes Portal vor, bei dem die Verfügbarkeit von Informationen in Designprojekten in Form von semantischen Framesets verarbeitet und gespeichert werden können. Unter Anwendung der vorgeschlagenen Modellierung wird die Definition eines Produkts sukzessiv demonstriert und Informationsverbindungen zwischen verschiedenen Teilergeb-nissen in Modulen (Prozesse, Funktionen, Effekte und Formen) abgelegt (Saue, Degenstein, Chahadi, Birkhofer, 2006). Zheng et al. schlagen für die Auswahl von additiven Fertigungsprozessen einen axiomatischen Designansatz vor, bei dem mit der Methode von gewichteten Präferenzdiagrammen personalisierte Evaluierungen eingeführt werden. Die Fuzzy-Mengen-Theorie wird übernommen, um qualitative Leistungsbewertungen von Materialien, Parametern und Zustände von Maschinen in deterministische Werte wie Kosten und Bauzeit zu wandeln (Zheng, Wang, Xu, Xie, 2017).

Generell kann zum jetzigen Stand Forschung festgehalten werden, dass eine Betrachtung aller für den Entwurf von additiv zu fertigende Flugzeug-strukturkomponenten relevanter Informationen sowie dessen Verzahnung aus den jeweiligen Prozessparametern, Konstruktions- bzw. Projektvorgaben und abgleitenden Designentscheidungen in Form eines virtuellen Beratungssystems mit einer adaptiven Informationsschnittstelle nicht verfügbar ist.

\section{Ziele und wissenschaftliche Fragestellungen}

Das primäre Ziel des Vorhabens ist es zu erforschen, inwiefern ein effektiver Grundstein eines interaktiven Gestaltungs- und Konstruktionsratgeber für additiv gefertigte Bauteile gelegt werden kann. Dieser soll Konstrukteuren zukünftig den schnellen und intuitiven Zugriff auf aktuelles und gesichertes Wissen für die optimale Formgestaltung und Dimensionierung von Flugzeugstrukturbauteilen geben. Für die Definition, 
Pflege und Weiterentwicklung soll ein Arbeitskreis, in dem Vertreter unterschiedlicher Organisationsformen der Luftfahrtindustrie mitwirken, die stetig konsensbasiert Expertenwissen und Erkenntnisse einbringen und die Wissensbasis auf Aktualität und Richtigkeit überprüfen.

Die Forschungsarbeit soll sich dabei mit den nachstehenden Fragestellungen im wissenschaftlichen Diskurs auseinandersetzen:

1. Welche Vorgehensweisen und wissenschaftlichen Methoden eignen sich für die disziplinübergreifende Entwicklung von VIKA?

2. Wie kann der beachtlichen Dynamik des Beratungsumfelds (Werkstoffe, Maschinen, Verfahren) Rechnung getragen werden?

3. Was sind luftfahrtspezifische Aspekte bei der Beratung zur additiven Fertigung und wie lässt sich dieses Wissen abbilden und vermitteln?

4. Wie kann ein virtueller Konstruktionsberater die heterogenen Wissensstände und Fragestellungen der Anwender berücksichtigen und jedem Nutzer eine individualisierte Antwort geben?

5. Wie kann proprietäres Wissen in der Anwendung geschützt abgebildet und an berechtigte Anwender ausgegeben werden und wie gelingt es, die Besitzer*innen dieses Wissens davon zu überzeugen, es dem System anzuvertrauen (Wissensschutz und Rollendefinition)?

6. Wie und womit lassen sich die Ergebnisse der Konzeptstudien softwaretechnisch realisieren?

\section{Entwurf und Ausarbeitung der Konzeptstudien}

Für die Ausarbeitung und Beantwortung der vorgestellten Forschungsfragen ist es notwendig, in einer geordneten Form das Vorhaben unter Einbezug des sogenannten "Systemdenkens" vorzugehen.

Bei dieser Methode zur Findung des Systemdesigns in Anlehnung an die VDI 2206 wird mit Hilfe einer Systemmodellierungssprache eine Formalisierung für Anforderungen, Systembeschreibung, Anwendungsfälle und Strukturdefinitionen vorgenommen und ein finales Systemdesign abgeleitet (VDI, 2206). 


\section{Globale Betrachtung}

Zunächst gilt es den Systemkontext zu erarbeiten. Dies erfolgte unter anderem über die oben formulierten Ziele und Fragen an das Vorhaben. Für diese Betrachtung ist es zudem erforderlich, die Systemgrenzen zu definieren und Schnittstellen festzulegen. Der Rahmen dieser Forschungsarbeit legt eine in sich geschlossene Konzeptstudie einer virtuellen Beratungsanwendung vor. (Eine) Schnittstelle(n) zu anderen Systemen und anderen Softwareanwendungen wird/ werden für die weitere Betrachtung ausgeschlossen. Für die Umsetzung stehen begrenzte Ressourcen zur Verfügung, die es für die Ausarbeitung zu berücksichtigen gilt. Für die spätere Verwertungsperspektive und zielorientierte Nutzung der Anwendung wird ein abweichender Bearbeitungskontext erforderlich.

\section{Funktionale Sicht}

Die im obigen Abschnitt aufgezeigten Voruntersuchungen liefern einen Anstoß für weitere Forschungsanstrengungen, eine Morphologie für konzeptionelle Umsetzungen zu diskutieren. Die funktionale Betrachtung des Systems liefert Möglichkeiten das Systemverhalten und seine funktionalen Abläufe weiter zu spezifizieren. Dabei werden wissenschaftliche Betrachtungen in Erwägung gezogen, die Anwender-System- Interaktionen und Funktionsstrukturen für VIKA aus dem Persona-Cluster (vgl. Abschnitt 2) zu extrahieren. Die Funktionen wurden kategorisiert und in 15 priorisierte Hauptfunktionen mit abgeleiteten Neben- bzw. Unterfunktionen überführt bzw. ergänzt (siehe Abbildung 4).

Aus den Funktionen konnte in Anlehnung an die VDI 2206 eine umfangreiche Anforderungsliste für die Erarbeitung einer ersten Konzeptidee erzeugt werden. Für ein stimmiges Systemdesign wurde der Beratungsprozess mit der Methode des Brainstormings fokussiert untersucht und zehn Möglichkeiten wurden identifiziert. Für wissenschaftliche Diskussion wurde mit Hilfe eines morphologischen Kastens die SystemDesign-Konzepte gegenübergestellt und unter den Aspekten „Realisierbarkeit“, „Erfüllungsgrad der angedachten Systemfunktionen“, „innovatives Potential“, „Benutzerakzeptanz" und "Verwertungsperspektive“ bewertet. Resultierend wurden zwei Varianten näher betrachtet. Aus der Untersuchung ging hervor, dass eine sprachgesteuerte dialogische Anwendung als Störfaktor keine breite und erforderliche Nutzerakzeptanz finden wird.

Als sehr innovativ wurde der dialogisch textuelle Assistent bewertet. Eine virtuelle Lösung in Form eines Agenten/Avatars/Bots basierend auf künstlicher Intelligenz und 
maschinellen Lernens könnte ohne eine spezifische CAx-Systemschnittstelle die Anwender in der täglichen Konstruktionsarbeit signifikant unterstützen. „Solche Lösungen lassen sich ideal auf eine abgegrenzte Wissensbasis applizieren. Jedoch befinden sich diese Anwendungen überwiegend noch in einem unausgereiften Stadium und fördern oftmals das Frustpotential der Anwender" (Kabel, 2019).

\begin{tabular}{|c|c|c|c|c|}
\hline \multicolumn{2}{|c|}{ Mögliche Primärfunktionen } & \multicolumn{2}{|c|}{ Mögliche Teilfunktionen } & \multirow{2}{*}{$\begin{array}{l}\text { Persona } \\
\text { Anzahl }\end{array}$} \\
\hline $\mathrm{Nr}$. & Funktion & Nr. & Funktion & \\
\hline \multirow[t]{7}{*}{5.} & \multirow{7}{*}{$\begin{array}{l}\text { Verbesserungsvorschläge } \\
\text { bei Konstruktion } \\
\text { bereitstellen }\end{array}$} & 5.1 & Verbesserungsvorschläge machen & 3 \\
\hline & & 5.2 & Auf Fehler aufmerksam machen & 1 \\
\hline & & 5.3 & Auf Problembereiche hinweisen & - \\
\hline & & 5.4 & Anpassung bestehender Bauteile vorschlagen & 1 \\
\hline & & 5.5 & Thermischen Verzug vermeiden & 1 \\
\hline & & 5.6 & Schrumpfung kompensieren, Wrapping vermeiden & - \\
\hline & & 5.7 & $\begin{array}{l}\text { Optimierungspotenziale \&Unsicherheiten } \\
\text { erkennen (Topologie/Shape) }\end{array}$ & 2 \\
\hline \multirow[t]{3}{*}{6.} & \multirow{3}{*}{$\begin{array}{l}\text { Konstruktion überprüfen } \\
\text { (CAD-Datensatz) }\end{array}$} & 6.1 & Geometrie für die Fertigung aufbereiten & \\
\hline & & 6.2 & CAD-Daten auf Vollständigkeit/Fehler überprüfen & 8 \\
\hline & & 6.3 & $\begin{array}{l}\text { Einhaltung von Gestaltungsrichtlinien unterstützen } \\
\text { (Mindestwand/Schichtdicke/Abstände/Überhänge) }\end{array}$ & 2 \\
\hline \multirow[t]{5}{*}{7.} & \multirow{5}{*}{$\begin{array}{l}\text { Druckprozess } \\
\text { unterstützen }\end{array}$} & 7.1 & Optimale Orientierung im Bauraum anzeigen & 1 \\
\hline & & 7.2 & Stützstruktur anzeigen/hinzufügen & - \\
\hline & & 7.3 & Menge an Stützstruktur anzeigen & - \\
\hline & & 7.4 & $\begin{array}{l}\text { Materialbedarfs/Kosten kalkulieren } \\
\text { Produktionszeit abschätzen }\end{array}$ & 5 \\
\hline & & 7.5 & Notwendige Nachbearbeitung anzeigen & - \\
\hline \multirow[t]{4}{*}{8.} & \multirow{4}{*}{$\begin{array}{l}\text { AM-Verfahren } \\
\text { empfehlen }\end{array}$} & 8.1 & AM-Verfahren empfehlen & 3 \\
\hline & & 8.2 & Eignung von AM-Verfahren anzeigen & 1 \\
\hline & & 8.3 & Vorteile zu AM-Verfahren aufzeigen & 1 \\
\hline & & 8.4 & Benchmarking ermöglichen & - \\
\hline \multirow[t]{4}{*}{9.} & \multirow{4}{*}{$\begin{array}{l}\text { Hilfestellung } \\
\text { ermöglichen } \\
\text { (Support, Kontakt }\end{array}$} & 9.1 & Hinweise einblenden & 2 \\
\hline & & 9.2 & FAQ beantworten & - \\
\hline & & 9.3 & $\begin{array}{l}\text { Kontaktaufnahme (Ansprechpartner, } \\
\text { Expertenanfrage) ermöglichen }\end{array}$ & 5 \\
\hline & & 9.4 & Level basierte Antworten erhalten & 1 \\
\hline
\end{tabular}

Abbildung 4: Auszug aus der Persona- Funktionsanalyse

Dennoch galt es für einen ersten Konzeptansatz diese Variante nicht kategorisch auszuschließen. Mit der Szenario-Technik wurde ein „User-Journey“ mit einer selektierten Persona entworfen, indem beispielhaft eine konkrete Konstruktions-aufgabe ausgewählt und dialogisch aufbereitet wurde. Der Dialog umfasste dabei die Interaktion zwischen dem Anwender und dem System ohne dabei Bezug auf die technische Realisierung zu nehmen. Die erste Konzeptidee wurde einem ausgewählten Auditorium am Zentrum für angewandte Luftfahrtforschung als Anstoß vorgestellt und kritisch diskutiert. Resultierend wurde bemerkt, dass für eine spätere Umsetzung, Wartung und 
Pflege eines solchen virtuellen Beratungsagenten nicht verfügbare Ressourcen notwendig seien. Somit wurde der Konzeptvorschlag kategorisch ausgeschlossen.

Die Vertreter aus der Luftfahrtindustrie wurden dahingehend befragt, welche Funktionalitäten aus ihrer Sichtweise in einer virtuellen Anwendung von großer Relevanz seien (siehe Abbildung 5).

$$
(n=15)
$$

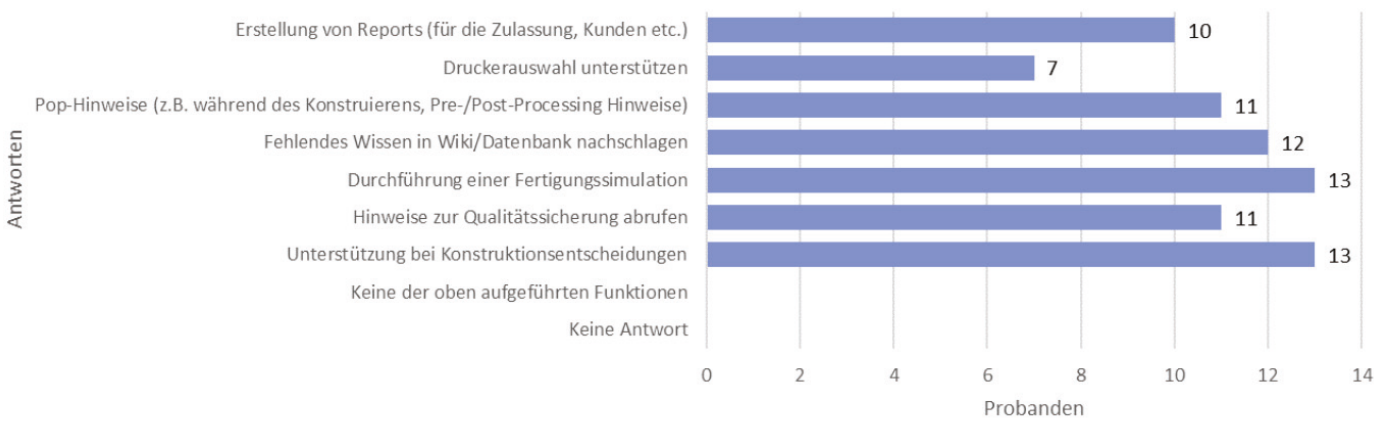

Abbildung 5: Auszug: Abfrage von relevanten Funktionen in VIKA

Über die in Abbildung 6 vorgeschlagenen Repräsentationsformen von Wissen wurde abgeleitet, dass sich eine webbasierte Plattform mit administrativ verteilten Anwenderrollen als eine zielorientierte Lösung anbietet, um das implizite Wissen für Konstruktionsmethodik und Herangehensweise in der additiven Fertigung in Form von Videos und Anwenderleitfäden als auch Anleitungsdokumente aufzubereiten.

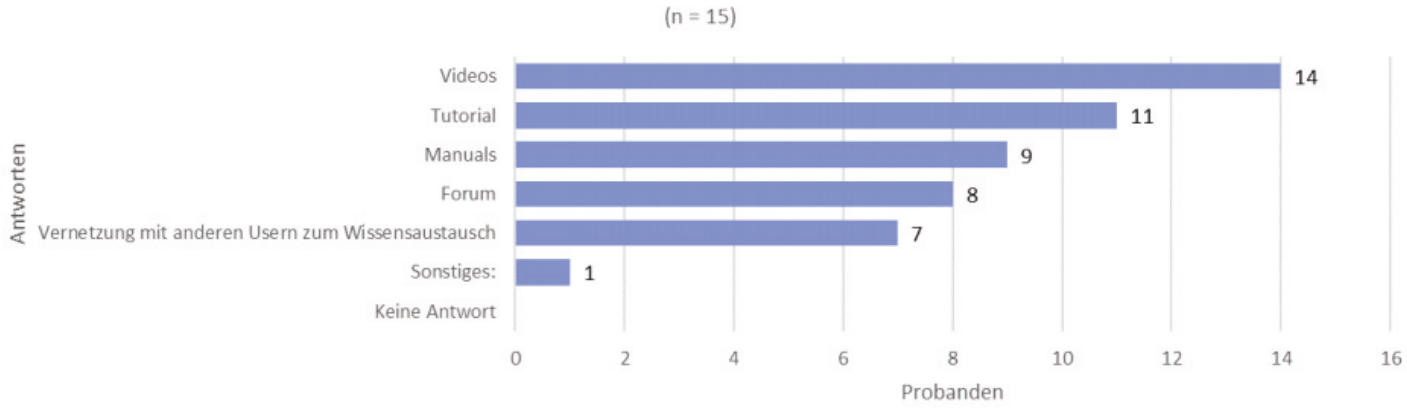

Abbildung 6: Auszug: Abfrage - favorisierte Möglichkeiten zur Wissensrepräsentation 
Zudem gilt es die Vernetzung der Anwender untereinander zu fördern, damit ein konstruktiver Wissensaustausch vorgenommen werden kann. Die Anwendung soll Möglichkeiten bieten, Konstruktionsentscheidungen dialogisch zu begleiten und die getroffene Auswahl in eine adaptiv angepasste Wissensrepräsentationsform zu überführen. Die Wege zwischen den Fragen an das System zu den bereitgestellten Informationen sind möglichst kurz zu halten, sodass eine effektive Unterstützung im Produktentstehungsprozess ermöglicht wird. Dabei soll sich VIKA deutlich von statisch virtuellen Repräsentationsformen abgrenzen.

\begin{tabular}{|c|c|c|c|c|c|c|c|c|c|c|c|c|c|c|c|}
\hline Einfluss von & $M$ & 0 & BG & G & GO & B & K & $\mathbf{F}$ & $\mathrm{TH}$ & PZ & $A B$ & w & PP & $\begin{array}{l}\text { Aktivsumme } \\
\text { AS }\end{array}$ & $\begin{array}{l}\text { Quotient } \\
\text { (AS/PS) }\end{array}$ \\
\hline $\begin{array}{c}\text { Material } \\
\mathbf{M}\end{array}$ & & 3 & 2 & 3 & 1 & 3 & 2 & 3 & 3 & 3 & 1 & 3 & 3 & 30 & 1,25 \\
\hline $\begin{array}{c}\text { Oberfläche } \\
\text { o }\end{array}$ & 0 & & 0 & 3 & 0 & 3 & 0 & 3 & 3 & 3 & 0 & 3 & 3 & 21 & 1,24 \\
\hline $\begin{array}{c}\text { Bauteilgröße } \\
\text { BG }\end{array}$ & 3 & 0 & & 0 & 2 & 0 & 2 & 0 & 3 & 3 & 3 & 3 & 2 & 21 & 1,91 \\
\hline $\begin{array}{c}\text { Genauigkeit } \\
G\end{array}$ & 0 & 0 & 0 & & 2 & 0 & 3 & 3 & 3 & 3 & 0 & 2 & 3 & 19 & 1,19 \\
\hline $\begin{array}{l}\text { Geometrie } \\
\text { GO }\end{array}$ & 3 & 1 & 0 & 0 & & 0 & 2 & 3 & 3 & 3 & 3 & 2 & 2 & 22 & 1,57 \\
\hline $\begin{array}{c}\text { Beständigkeit } \\
\text { B }\end{array}$ & 3 & 1 & 0 & 0 & 0 & & 3 & 3 & 1 & 0 & 0 & 0 & 1 & 12 & 1,09 \\
\hline $\begin{array}{c}\text { Konstruktionsziel } \\
\mathrm{K}\end{array}$ & 3 & 3 & 3 & 1 & 2 & 2 & & 3 & 3 & 2 & 2 & 2 & 2 & 28 & 1,12 \\
\hline $\begin{array}{c}\text { Funktionalität } \\
\text { F }\end{array}$ & 3 & 3 & 1 & 3 & 3 & 1 & 3 & & 3 & 0 & 0 & 0 & 2 & 22 & 0,88 \\
\hline $\begin{array}{c}\text { Tech. Herstellbarkeit } \\
\text { TH }\end{array}$ & 2 & 3 & 2 & 3 & 2 & 0 & 3 & 3 & & 1 & 3 & 2 & 2 & 26 & 1,00 \\
\hline $\begin{array}{l}\text { Produktionszeit pro Stück } \\
\text { PZ }\end{array}$ & 1 & 0 & 0 & 0 & 0 & 0 & 2 & 0 & 1 & & 2 & 3 & 1 & 10 & 0,48 \\
\hline $\begin{array}{c}\text { Ausnutzung Bauraum } \\
\mathrm{AB}\end{array}$ & 1 & 0 & 0 & 0 & 0 & 0 & 0 & 0 & 0 & 0 & & 0 & 0 & 1 & 0,06 \\
\hline $\begin{array}{c}\text { Wirtschaftlichkeit } \\
\text { w }\end{array}$ & 2 & 0 & 0 & 0 & 0 & 0 & 3 & 1 & 1 & 0 & 3 & & 0 & 10 & 0,45 \\
\hline $\begin{array}{l}\text { Post-Processing } \\
\text { Pp }\end{array}$ & 3 & 3 & 3 & 3 & 2 & 2 & 2 & 3 & 2 & 3 & 1 & 2 & & 29 & 1,38 \\
\hline $\begin{array}{c}\text { Passivsumme } \\
\text { PS }\end{array}$ & 24 & 17 & 11 & 16 & 14 & 11 & 25 & 25 & 26 & 21 & 18 & 22 & 21 & \multirow{2}{*}{\multicolumn{2}{|c|}{$\begin{array}{l}\text { 0: kein Einfluss } \\
\text { 1: geringer Einfluss } \\
\text { 2: mittlerer Einfluss } \\
\text { 3: starker Einfluss }\end{array}$}} \\
\hline Produkt (AS*PS) & 720 & 357 & 231 & 304 & 308 & 132 & 700 & 550 & 676 & 210 & 18 & 220 & 609 & & \\
\hline
\end{tabular}

\section{Abbildung 7: Wechselwirkungsmatrix - Verfahrensempfehlungen}

Zudem zeigte die Auswertung der Umfrage, dass ein sehr großer Bedarf bei der gänzlichen Prozessberatung existent sei. Dafür wurde im nächsten Schritt ein semantisches Netz für die Prozessselektion additiv herzustellender Bauteile erzeugt, um wichtige Entscheidungskriterien zu identifizieren. Mit einer Wechsel-wirkungsmatrix wurden die erarbeiten Parameter verglichen und es konnte festgestellt werden, dass die Materialauswahl, die Anforderungen an die Oberfläche als auch die Bauteilgröße treibende Faktoren für alle folgenden Entscheidungen im Prozess sowie in der Produktion bilden (siehe Abbildung 7). Es sei jedoch zu erwähnen, dass die Auswertung sehr stark subjektiv getrieben und somit kritisch zu hinterfragen sei. Aus der Fachliteratur geht 
hervor, dass die Entscheidungsfindung im gänzlichen Bauteilentstehungs- und Fertigungsprozess als hochkomplex einzustufen ist.

Um fallbasierte Entscheidungen virtuell zu unterstützen, kommen oftmals regelbasierte Expertensysteme zum Einsatz, die auf Basis einer erweiterbaren Wissensbasis Aktionen über implementierbare Eingaben der Anwender ausführen. Aus der Literatur konnte entnommen werden, dass sehr viele dieser XPS-Systeme nicht über das Prototypenstadium hinausgekommen sind, da die Aufwände für die spätere Pflege und Wartung der Systeme in keinem Verhältnis zum eigentlichen Nutzen standen (Hartmann 2015). Für eine Umsetzung eines effektiven XPS-Systems sind zudem über einen größeren Zeitraum Kapazitäten von disziplinübergreifenden Experten zu binden, die im Zusammenwirken mit Software- und Knowledge-Ingenieuren das impliziten Wissen in Regeln und Algorithmen transformieren, um es für die Anwender übersetzt nützlich zu machen. Dieser Umstand würde den Rahmen dieses Forschungsvorhabens sprengen und wird im Sinne der Verwertungs-perspektive nicht weiterverfolgt.

\section{Strukturbetrachtung}

Auf Basis der Voruntersuchungen und modellierten Interaktionssequenzen mit dem System gehen folgende Systembausteine für eine weitere prototypentechnische Umsetzung hervor:

- ein Modul zur dialogischen Konstruktionsberatung

- ein Modul für explorative Suchvorgänge von Begriffen und Ontologien

- ein virtuelles Nachschlagewerk (Wiki)

- ein Modul für die administrative Benutzerkonten- und Projektverwaltung

- ein Modul für die Vernetzung der Anwender (Foren, Mitteilungen)

- eine Unterstützungsschnittstelle (FAQ, Kontakt)

- eine Datenbank (Backend) für adaptive Wissensrepräsentation auf der Grundlage einer erweiterbaren und editierbaren Wissensbasis

Eine Lücke in der Forschung wurde in der ganzheitlichen Prozessberatung dahingehend identifiziert, dem Anwender die semantischen Zusammenhänge und Wechselwirkungen der Prozessbausteine und -parameter anhand einer interaktiven und virtuellen Anwendung mit gestuften Informationenebenen zu präsentieren. In einer solchen Anwendung könnte im Frontend einer Webanwendung die Erzeugung von sogenannten Ansichtsebenen, die über Aggregation und „Details on Demand“ im „Intra- 
Level change“ nach Gundelsweiler organisiert sind, eine adaptive Wissensrepräsentation dem Benutzer vorgeschlagen werden (Gundelsweiler, Memmel, Reiterer 2009). Dafür gilt es eine mehrstufige Struktur der Systembausteine und deren Vernetzung zu erarbeiten (siehe Abbildung 8). Diese Strukturen werden in der Detaillierung nach dem angesprochenen Top-Down-Prinzip ausgearbeitet und auf Plausibilität überprüft.

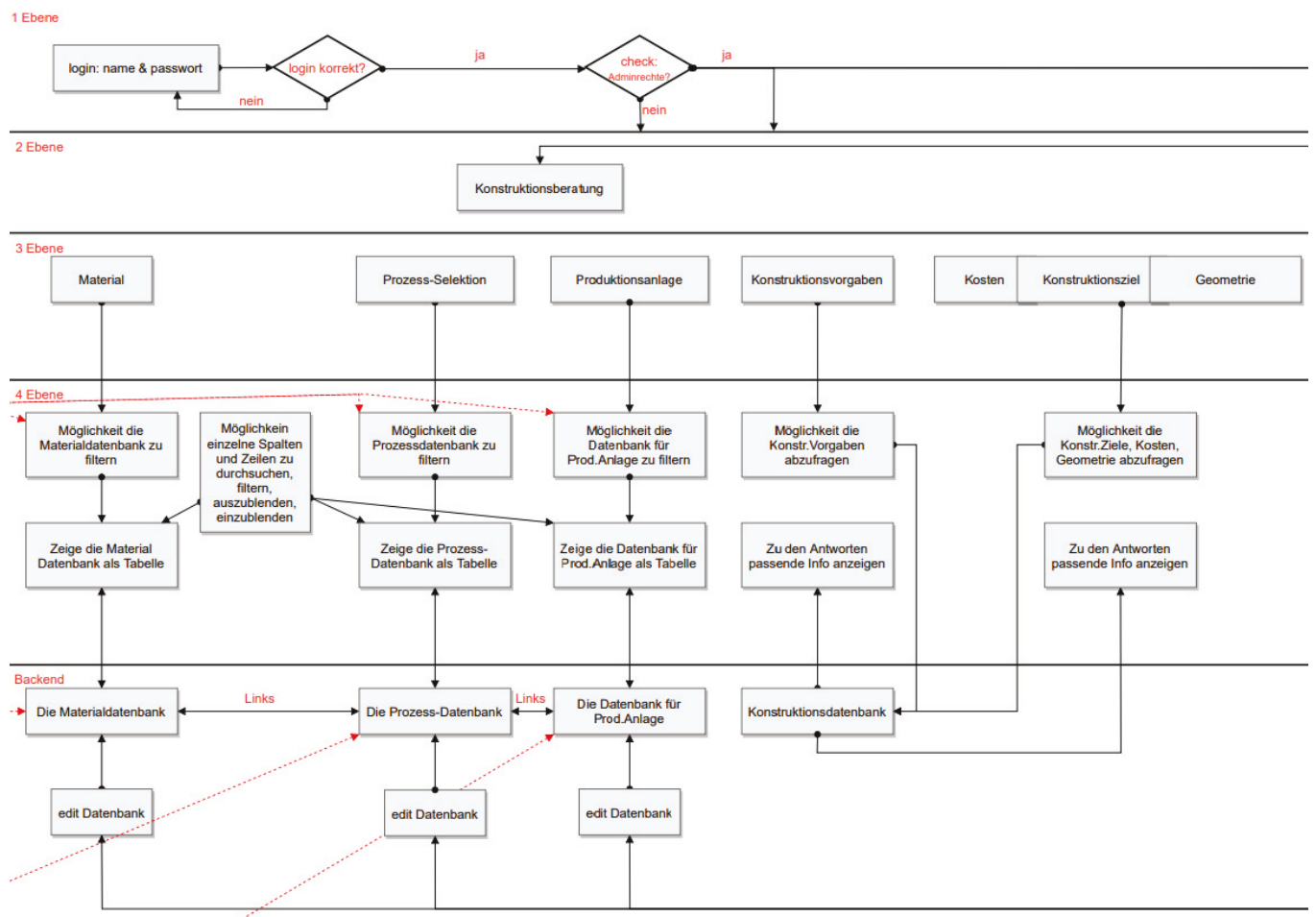

Abbildung 8: Auszug: Vernetzung der System- und Funktionsbausteine

\section{Weiteres Vorgehen und Ausblick}

Für die weitere Ausarbeitung ist es unabdingbar, die systematisch aufbereiten Inhalte zu indizieren, sodass eine logische Verknüpfung mit der Datenbank gelingt. Die Datenbank verwaltet die Freitextsuche und aggregiert die abgebildete Wissensbasis in der Informationsdarstellung im Frontend auf Basis von zulässigen Eingabeparametern oder angebotene Auswahlmöglichkeiten. 
Es sei zu erwähnen, dass eine virtuelle Ausarbeitung mit sogenannten „Open-Source“Lösungen betrachtet wird. Die weitere Modellierung der Wissensstrukturen und der Systembausteine wird zunächst mit grafisch basierten Softwarelösungen (UML und SYS ML) vorgenommen. Nachdem eine ausführliche Bewertung der verfügbaren Softwarelösungen zur Umsetzung vorgenommen ist, können Front- und Backend aufgebaut werden, um einen Grundgerüst für die Weiterentwicklung von VIKA zu schaffen. Dieses Vorgehen soll durch Anwendertest und Befragungen begleitet werden, um Verbesserungen in das Systemdesign einfließen zu lassen. Zudem wird eine Systematik und Strategie erarbeitet, um zukünftig implizites Expertenwissen aus der Luftfahrtindustrie in den Berater zu implementieren. Dafür soll ich im Nachgang zum Vorhaben VIKA eine Arbeitsgemeinschaft gründen, die zukünftig diese Aufgaben übernimmt.

\section{Literaturverzeichnis}

Agarwala, R. \& Chin, R. A. (2015): Facilitating Additive Manufacturing Engagement and Outreach, ASEE annual conference and exposition, Seattle, Washington. 1018260/p.24086

ASTM Comitee F42 on additive manufacturing technologies (2009): ASTM F2792-10 standard terminology for AM technologies, West Conshohocken, PA Cooper, Reimann, Cronin, 2007

Eisman, E. M. \& Lòpez, V. \& Castro, J. L. (2012): A framework for designing closed domain virtual assistants. In: Expert Systems with Applications, 39 (3), 3135-3144

Gundelsweiler, F. \& Memmel, T. \& Reiterer, H. (2009): ZUI Konzepte für Navigation und Suche in komplexen Informationsräumen (ZUI Concepts for Navigating and Searching Complex Information Spaces). In: i-com, 6 (1), 38-47

Hartmann, K. (Hrsg.) (2015): Einführung in die Expertensystem-Technologie, Merseburg, Hochschulverlag Merseburg Helmrath, C. \& Catarino, P. \& Brackett, D. (2020): Encompass - gefördert durch European Union's Horizon 2020: Beitrag im Internet. http://encompass-am.eu, abgerufen am 21.10.2020

Kabel, P. (2019): Experteninterview. In: tell-me days 2019 Fachkonferenz an der HAW Hamburg, Fakultät Design Medien und Informationen, Hamburg

Kratzer, M. (2014): Anwendungsspezifische Entwicklung eines proaktiven Konstruktionssystems auf Basis von Softwareagenten. In: Bericht / KTD, Institut für Konstruktionstechnik und Technisches Design, Stuttgart

Luft, T. \& Breitsprecher, T. \& Roth, D.\& Lindow, K. \& Wartzack, S.\& Binz, H. (2012): Die Rolle des Wissensingenieurs im Unternehmen - Ergebnisse einer Umfrage und Darstellung in der VDI-Richtlinie "Wissensbasiertes Konstruieren". In: Wartzack, S.\& Krause, D. \& Paetzold, K. (Hrsg.): Design for X: Beiträge zum 23. DfX-Symposium Oktober 2012. Hamburg: TuTech Verlag, S.63-78

Pahl, G. \& Beitz, W. \& Feldhusen, J. \& Grote, K.-H. (Hrsg.) (2007): Engineering Design: A Systematic Approach, London, Springer-Verlag

Qi, Q. \& Pagani, L. \& Scott, P. J. \& Jiang, X. (2018): A categorical framework for formalising knowledge in additive manufacturing. In: Procedia CIRP, 75, 87-91 
Remus, U. (2002): Prozessorientiertes Wissensmanagement. Konzepte und Modellierung, Regensburg, epub.uni-regensburg.de

Saue, T. \& Degenstein, T. \& Chahadi, Y. \& Birkhofer, H. (2006): A Web-based Information Portal for the early stages of design. In: Proceedings Design 2006, the 9th International Design Conference, Dubrovnik, Croatia

Serdar, T. (2016): Educational Challenges in Design for Additive Manufacturing. In: ASEE, New Orleans, Lousiana. $10.18260 / p .27294$

VDI-Gesellschaft Produkt- und Prozessgestaltung (Hrsg.) VDI 2206 (2004): Ent-wicklungsmethodik für mechatronische Systeme, Düsseldorf, VDI-Verlag

Wang, Y. \& Zhong, R. Y. \& Xu, X. (2018): A decision support system for additive manufacturing process selection using a hybrid multiple criteria decision-making method. In: Rapid Prototyping Journal, 24 (9), 1544-1553

Yao, X. \& Moon, S. K. \& Bi, G. (2017): A hybrid machine learning approach for additive manufacturing design feature recommendation. In: Rapid Prototyping Journal, 23 (6), 983-997

Zheng, P. \& Wang, Y. \& Xu, X. \& Xie, S. Q. (2017): A weighted rough set based fuzzy axiomatic design approach for the selection of AM processes. In: The International Journal of Advanced Manufacturing Technology, 91 (5-8), 1977-1990

\section{Kontakt}

Johann Steffen, M. Sc.

HAW Hamburg

Berliner Tor $7 a$

20099 Hamburg

www.haw-hamburg.de

Johann.Steffen@haw-hamburg.de 